\title{
Improving physician's adherence to completing vaccination schedules for patients with type 2 diabetes attending non-communicable diseases clinics in West Bay Health Center, Qatar
}

\author{
Hassan Tawfik, Zelaikha Bashwar, Amal Al-Ali, Mohamed Salem, Isameldin Abdelbagi \\ Primary Health Care Corporation(PHCC) Qatar
}

\begin{abstract}
Incomplete vaccination for patients with type 2 diabetes attending non-communicable diseases (NCD) clinics is an issue that could affect patient's health and wellness negatively and puts patients at high risk of serious diseases.
\end{abstract}

We aimed to improve physicians adherence to complete vaccination schedule for patients with type 2 diabetes attending NCD clinics in west bay health center according to American Diabetes Association (ADA) recommendation by 25\% by January 2015.

In the pre-intervention phase: the quality improvement team designed a checklist to collect the percentage of physician's adherence of prescription of the recommended vaccination for patients with type 2 diabetes. The percentage of complete vaccination in patients with diabetes attending NCD clinic in West Bay Health Center was $20 \%$.

In the intervention phase the intervention was in the form of: the creation a vaccination form and attached to the (NCD) progress note; to distribute and remind the physicians about the ADA guidelines vaccination recommendations; a summary of the vaccination schedule developed and attached to (NCD) form; development of vaccination reminder posters and posters in the waiting area, nurse station, and physician clinics and education and orientation sessions for NCD clinic staff.

In the post-intervention phase the average percentage of complete vaccination in patients with diabetes attending NCD clinic in West Bay Health Center increased to $69 \%$.

\section{Problem}

The rationale of recommendation of use of influenza and pneumococcal vaccine in people with diabetes by American Diabetes Association technical review is due to the abnormalities in immunity and increased morbidity and mortality from infections in patients with diabetes.

Studies show that diabetics are in high risk for complications, hospitalization, and death from influenza and pneumococcal disease.[1] patients with diabetes should receive routine vaccinations as for all population.[2]

In spite influenza and pneumonia are common, there are limited studies reporting the morbidity and mortality of influenza and pneumococcal pneumonia specifically in people with diabetes. Observational studies of patients with a variety of chronic illnesses, including diabetes showed increase in hospitalization in population with chronic diseases, people with diabetes are at increased risk for bacteremia from pneumococcal infections with higher mortality rate as $50 \%$.[3]

Moreover, in a case control series influenza vaccine was show to reduce diabetes related hospital admission by as much as $79 \%$ during flu epidemics.[4]
Improper physicians adherence to internationally vaccine recommendations for patients with type 2 diabetes attending NCD clinics at West Bay Health Center will affect the quality of life and services provided to the target patients, especially when there is no clear data about percentage of complete vaccinations for patients with diabetes in Qatar.

\section{Background}

Patients with diabetes have good humoral immune responses to vaccination. Immunization against influenza and pneumococcal disease is an important part of preventive services for many chronic diseases such as diabetes.

According to the Advisory Committee on Immunization Practices (ACIP), the American College of Physicians, the American Academy of Pediatrics, and the American Academy of Family Physicians, vaccinating individuals at high risk before influenza season each year is the most effective measure for reducing the impact of influenza.[5]

The ADA endorses a recent CDC advisory panel that recommends administration of both PCV13 and PCV23 should be routinely in series to all adults 65 years of age or older.[6] 
Our aim is to improve physician adherence to complete vaccination schedule for patients with type 2 diabetes attending NCD clinic in West Bay health center by $25 \%$ by the January 2015 according to the ADA recommendation. The purpose of this study is to raise the physicians' awareness and improve the preventive services given to community.

\section{Baseline measurement}

The project began on the 21st of November 2014 with baseline data collection. This was conducted to determine the percentage of completed vaccination done on 22nd of November 2014, with the results being $20 \%$ in October and $0 \%$ November 2014 respectively. See figure 1 for more on which brainstorming session was done to identify the root causes of such problem, and depicted using the fish bone diagram featured there.

A survey questionnaire was designated to assess the main causes of the problem and distributed to nurses and physicians working in NCD clinics (table 1)

Prioritization of causes demonstrated in Paretto chart (figure 2). According to analysis we found three main causes of the problem:

1. Lack of awareness of physicians regarding the recommended vaccinations

2. No specific form or item in the NCD progress note about vaccinations

3. That physicians forget to document the vaccine that he prescribed.

See supplementary file: ds5590.docx - "Figures and Tables"

\section{Design}

In the pre-intervention phase: The data were collected retrospectively for the previous two months by using the checklist to show average percentage of vaccine documentation.

For NCD nurses the education session was done to particularly cover orientation about the new NCD format and vaccination documentation.

For NCD physicians: A vaccination form was created and attached to the non-communicable disease (NCD) progress note (see table 2 ), and orientation sessions were done for NCD clinic physicians. In addition to the distribution and reminders for the physicians about the ADA guideline vaccinations recommendations, a summary of the vaccination schedule was also developed and attached to vaccination form. As well as this, vaccination reminder posters were developed and placed in the waiting area, nurse station, and physician clinics (figure 3 ).

In the post-intervention phase: Same data collection tool in preintervention phase used to show the average percentage in postintervention stage ending at 15th January 2015. Two audits were conducted to assess vaccination coverage among patients with diabetes in April and May 2015 and we take average percentage for complete vaccination status

\section{Strategy}

PDSA cycle 1: Nurses' education and physician orientation was completed, showing a great

improvement.

PDSA cycle 2: Putting fixed item in the assessment and plan for vaccination in medical records.

PDSA cycle 3: New staff joined the clinic (physicians and nurses), so orientation regarding vaccination schedule was done.

Two audits conducted in April and May 2015 to assure sustainability. The health center will adopt the Cerner (electronic health record) so there was not much interest in completing the paper medical records. This project is to be continued by another quality improvement team who will be putting into consideration these problems; they will measure compliance and subsequent management, and it will be distributed to all health centers.

\section{Results}

In post intervention phase: The same data collection tool in preintervention phase was used to show the average percentage in post-intervention stage, ending at 15th January 2015 and all data were demonstrated using a run chart (figure 4).

The intervention was very successful in improving the percentage of complete vaccination in patients with type 2 diabetes attending NCD clinic in West Bay Health Center. In the pre-intervention the percentage was $20 \%$ and increased to $69 \%$ after the intervention.

See supplementary file: ds5963.docx - "Figure (4)"

\section{Lessons and limitations}

We learned a number of lessons from this project:

- Doing minor changes can cause major changes

- Education is one of the most powerful ways to influence health care adherence to recommended vaccinations

- Sharing physician and nurses in teamwork to achieve goals is very important for patient outcomes.

Sustainability is a very important consideration when you are concerned with achieving the goals of any improvement project. Sustainability will be ensured through same team continuous audit and the support of another team to continue the same project on electronic medical records through recently applied Cerner system.

The availability of vaccines is a major issue for success of such 


\section{BMJ Quality Improvement Reports}

interventions since in the second month of data collection the percentage increased by only $1 \%$ due to unavailability of pneumococcal vaccine.

\section{Conclusion}

An intervention in the form of education and orientation sessions for nurses and physicians, developing a vaccination form, attaching this to NCD progress note (to increase the awareness of the recommendations for complete vaccination), and the creation of an enhanced reminder system by developing vaccination reminder posters in the waiting area, nurse station and physician clinic all led to an improvement in vaccination percentage from $20 \%$ to $69 \%$.

\section{References}

1. Influenza and Pneumococcal Immunization in Diabetes (diabetes care, volume 27, supplement 1, January 2004)

2. Akinsanya-Beysolow I; Advisory Committee on Immunization Practices (ACIP); ACIP Child/Adolescent Immunization Work Group; Centers for Disease Control and Prevention (CDC). Advisory Committee on Immunization Practices recommended immunization schedules for persons aged 0 through 18 years - United States, 2014. MMWR Morb Mortal Wkly Rep 2014;63:108-9.

3. Smith SA, Poland GA. Use of influenza and pneumococcal vaccines in people with diabetes. Diabetes Care 2000;23:95-108.

4. Colquhoun AJ, Nicholson KG, Botha JL, Raymond NT. Effectiveness of influenza vaccine in reducing hospital admissions in people with diabetes. Epidemiol Infect 1997;119:335-41.

5. Bridges CB, Coyne-Beasley T; Advisory Committee on Immunization Practices (ACIP); ACIP Adult Immunization Work Group; Centers for Disease Control and Prevention (CDC). Advisory Committee on Immunization Practices recommended immunization schedule for adults aged 19 years or older - United States, 2014. MMWR Morb Mortal Wkly Rep 2014;63:110-2.

6. Tomczyk S, Bennett NM, Stoecker C, et al. Use of 13-valent pneumococcal conjugate vaccine and 23-valent pneumococcal polysaccharide vaccine among adults aged 65 years: recommendations of the Advisory Committee on Immunization Practices (ACIP). MMWR Morb Mortal Wkly Rep 2014;63:822-5

\section{Declaration of interests}

Nothing to declare.

\section{Acknowledgements}

We would like to thank: Mrs: Hanan Raslan, staff nurse, Primary Health Care Corporation. the patient autonomy was preserved as he can refuse to receive vaccinations also

confidentiality and privacy maintained and the work done by permission from concerned health authoritis. 